\title{
ARTICLE
}

\section{Active control of magnetoresistance of organic spin valves using ferroelectricity}

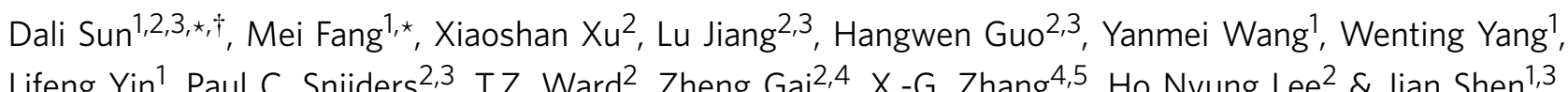

Organic spintronic devices have been appealing because of the long spin lifetime of the charge carriers in the organic materials and their low cost, flexibility and chemical diversity. In previous studies, the control of resistance of organic spin valves is generally achieved by the alignment of the magnetization directions of the two ferromagnetic electrodes, generating magnetoresistance. Here we employ a new knob to tune the resistance of organic spin valves by adding a thin ferroelectric interfacial layer between the ferromagnetic electrode and the organic spacer: the magnetoresistance of the spin valve depends strongly on the history of the bias voltage, which is correlated with the polarization of the ferroelectric layer; the magnetoresistance even changes sign when the electric polarization of the ferroelectric layer is reversed. These findings enable active control of resistance using both electric and magnetic fields, opening up possibility for multi-state organic spin valves.

\footnotetext{
${ }^{1}$ State Key Laboratory of Surface Physics and Department of Physics and Collaborative Innovation Center of Advanced Microstructure, Fudan University, Shanghai 200433, China. ${ }^{2}$ Materials Science and Technology Division, Oak Ridge National Laboratory, Oak Ridge, Tennessee 37831, USA. ${ }^{3}$ Department of Physics and Astronomy, University of Tennessee, Knoxville, Tennessee 37996, USA. ${ }^{4}$ Center for Nanophase Materials Sciences, Oak Ridge National Laboratory, Oak Ridge, Tennessee 37831, USA. ${ }^{5}$ Computer Science and Mathematics Division, Oak Ridge National Laboratory, Oak Ridge, Tennessee 37831 , USA. * These authors contributed equally to this work. † Present address: Department of Physics and Astronomy, University of Utah, Salt Lake City, Utah 84112, USA. Correspondence and requests for materials should be addressed to X.X. (email: xiaoshan.xu@unl.edu) or to J.S. (email: shenj5494@fudan.edu.cn).
} 
S

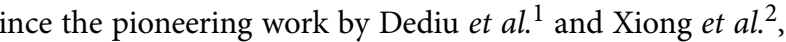
many of the follow-up studies have focused on achieving high magnetoresistance (MR) in organic spin valves (OSVs) and uncovering the underlying transport mechanisms $s^{3-11}$. It has been generally acknowledged that the interfaces between the organic layer and the ferromagnetic electrodes play a critical role in determining the actual spin-dependent transport mechanism ${ }^{12-15}$. Barraud et al. ${ }^{16}$ studied the spin transport of an OSV in which the thin organic layer serves as a tunnelling barrier as opposed to a diffusive spacer. A spin transport model describing the role of interfacial spin-dependent metal/organic hybridization on the amplitude and sign of the MR was put forward ${ }^{16,17}$. Recently, Schulz et al..$^{18}$ observed a reversal of the spin polarization of extracted charge carriers by introducing a thin interfacial permanent dipolar layer (LiF). This work indicates that the local electric dipole moment at the interface is important for MR, although in such a device the added dipolar layer plays only a passive role in terms of controlling MR.

In the following, we employ a ferroelectric (FE) thin interfacial layer between the organic semiconductor and the ferromagnetic electrode in OSV to achieve active control of MR. The material of choice for the $\mathrm{FE}$ layer is $\mathrm{PbZr}_{0.2} \mathrm{Ti}_{0.8} \mathrm{O}_{3}$ (PZT), which has a large polarization $\left(\sim 80 \mu \mathrm{C} \mathrm{cm}^{-2}\right)^{19}$. The PZT layer can induce strong interfacial dipoles and built-in electric field between the organic spacer layer and the ferromagnetic electrode. The interfacial dipole is switchable by external electric field, potentially allowing the control of the spin polarization of injected carriers in organic spintronics. We show that the resistance can be controlled by not only the spin alignment of the two ferromagnetic electrodes ${ }^{3}$, but also by the electric polarization of the PZT layer. The sign of MR changes when electric polarization of the PZT layer is reversed. This new tunability can be understood in terms of the change of relative energy level alignment between ferromagnetic electrode and the organic spacer caused by the electric dipole moment of the FE layer.

\section{Results}

Device structure and characterizations of the PZT films. Our fabricated OSV s consist a 65-nm Alq 3 (tris-(8-hydroxyquinoline) aluminum) layer sandwiched between a 5-nm-thick PZT layer epitaxially grown on a 30 -nm-thick $\mathrm{La}_{0.67} \mathrm{Sr}_{0.33} \mathrm{MnO}_{3}$ (LSMO) film-buffered $\mathrm{SrTiO}_{3}$ (STO) substrate, and a 10-nm-thick (nominal thickness) cobalt layer with gold capping. In this LSMO/PZT/Alq ${ }_{3} / \mathrm{Co}$ junction (FE-OSV), Co and LSMO serve as the top and bottom magnetic electrodes, respectively. The device structure is schematically shown in Fig. 1a. As discussed in the following, such kind of devices exhibit striking tunability, that is, both the amplitude and sign of MR are tunable due to the presence of the FE PZT.

After epitaxial growth of PZT on LSMO/STO, the PZT layer has a smooth surface with atomically flat terraces, as characterized by atomic force microscopy shown in Fig. 1b. This provides an ideal base for preparing a well-defined $\mathrm{Alq}_{3} / \mathrm{PZT}$ interface. The purpose of introducing the 5-nm-thick-PZT is to tune the energy level alignment between the $\mathrm{Alq}_{3}$ and LSMO layers because the polarization in PZT is switchable by applying an electric field. Therefore, the polarization reversal of the PZT is crucial. Here we have characterized the polarization reversal of the PZT films used in the FE-OSV devices with piezoelectric force microscopy (PFM). Figure 1c shows a PFM image of the PZT film with a part of the film poled by a conducting tip with $\pm 2.5 \mathrm{~V}$ relative to the LSMO bottom electrode. It shows that the polarization of the asgrown films is pointing 'up', and a clear reversal of polarization between 'up' and 'down' states can be created by sign reversal of the applied voltage. Figure 1d shows the voltage dependence of
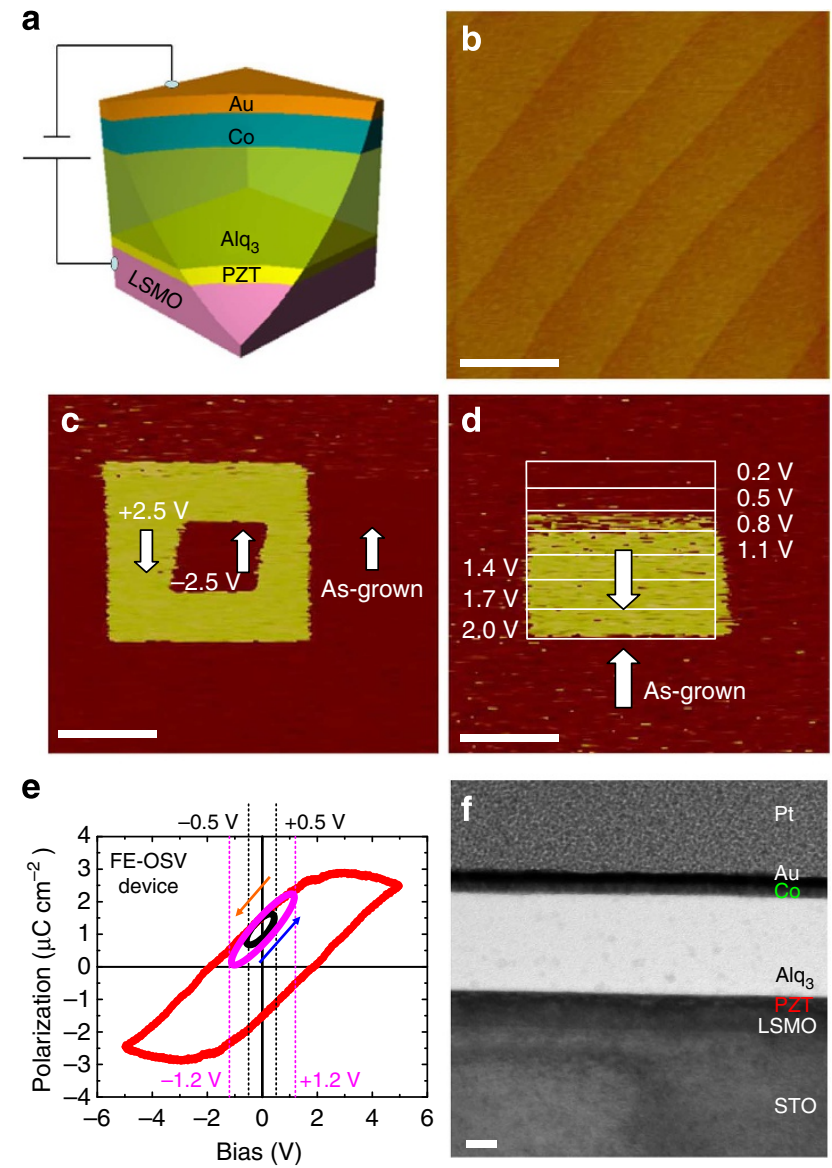

Figure 1 | Structure of the organic spin valves and characterization of ferroelectricity in the epitaxial PZT. (a) Schematic structure of a $\mathrm{Au} / \mathrm{Co}$ / $\mathrm{Alq}_{3} / \mathrm{PZT} / \mathrm{LSMO}$ organic spin valve (FE-OSV). (b) Atomic force microscopy topography image of a PZT layer ( $5 \mathrm{~nm}$ in thickness) epitaxially grown on a LSMO $(30 \mathrm{~nm}) / \mathrm{STO}$ substrate. (c) PFM (phase) response measured after successively switching the polarization of the PZT film by applying $+2.5 \mathrm{~V}$ and $-2.5 \mathrm{~V}$ on the tip with respective to the LSMO bottom electrode. Note that the protocol of voltage polarity is different in PFM measurements from that in resistance measurements. (d) PFM image showing the polarization reversal by gradually increasing the applied voltage. The 'up' ('down') arrow corresponds to the polarization pointing out of (into) the film surface. Scale bar, $500 \mathrm{~nm}$ (b-d). (e) A typical polarization-voltage loop for the FE-OSV (device A). The black and pink circles illustrate the 'minor loops' corresponding to $V_{\mathrm{MAX}}$ of \pm 0.5 and $\pm 1.2 \mathrm{~V}$, respectively. The orange (blue) arrow indicates the direction of the ramp voltage: sweeping down (up) before the MR scans. (f) TEM image for FE-OSV device. Different layers can be distinguished as labelled. Scale bar, $20 \mathrm{~nm}$.

the polarization. It is clear that the polarization of the PZT film ( $5 \mathrm{~nm}$ in thickness) starts to switch to the 'down' state when the voltage of the scanning probe exceeds $0.8 \mathrm{~V}$. The polarization reversal of the PZT in the FE-OSV is characterized by the measurements of the hysteretic polarization-voltage dependence. As shown in Fig. 1e, with the $\mathrm{Alq}_{3} / \mathrm{Co} / \mathrm{Au}$ layers on top of $\mathrm{PZT}$, the coercive voltage is $\sim 2 \mathrm{~V}$. Figure if shows the transmission electron microscope (TEM) image for the cross-section of the FEOSV. No significant diffusion of Co atoms into the $\mathrm{Alq}_{3}$ layer is observed (see Supplementary Fig. 1 and Supplementary Note 1).

Hysteretic behaviour of the MR. It has been known that MR depends sensitively on the measuring voltage $\left(V_{\mathrm{MR}}\right)$. Previous 
studies indicated that $\mathrm{MR}$ of $\mathrm{LSMO} / \mathrm{Alq}_{3} / \mathrm{Co}$ junctions (LSMOOSV) increases with decreasing $V_{\mathrm{MR}}$, reaching a maximum when $V_{\mathrm{MR}}$ is close to zero ${ }^{2,16}$. For our FE-OSV junctions, its MR depends not only on $V_{\mathrm{MR}}$, but also on the history of the voltage applied, giving rise to a strong hysteretic behaviour. We characterize this hysteretic behaviour by measuring $\mathrm{MR}\left(V_{\mathrm{MR}}\right)$ profile after applying a ramping voltage $\left(V_{\mathrm{MAX}}\right)$ that is larger than $V_{\text {MR }}$ (see Supplementary Fig. 2 and Supplementary Note 2 for the detailed measurement protocol).

As shown in Fig. 2, for a FE-OSV containing an as-grown PZT layer, the MR (at $T=11 \mathrm{~K}$ ) is always negative and is the largest when $V_{\mathrm{MR}}$ is near zero, which is similar to numerous previous studies on LSMO-OSV ${ }^{2,4-6,8-11}$. The different features in the FE-OSV are: (1) the $\mathrm{MR}\left(V_{\mathrm{MR}}\right)$ profile is strongly affected by the initial voltage $\left(V_{\mathrm{MAX}}\right)$ and (2) a positive (negative) $V_{\mathrm{MAX}}$ leads to a negative (positive) shift of the $\operatorname{MR}\left(V_{\mathrm{MR}}\right)$ profile along the $V_{\mathrm{MR}}$ axis (Fig. 2d,e). This shift $(\Delta V)$ is closely tied to the hysteretic behaviour of the PZT layer (so-called minor loops, see Fig. 1e) and increases with increasing $V_{\mathrm{MAX}}$, as shown in Fig. 2f. The switching fields, MR uncertainty and MR loops reproducibility in FE-OSVs are shown in Supplementary Figs 3-5 and Supplementary Notes 3 and 4, respectively.

The hysteretic behaviour of the MR was not observed in OSVs without the $\mathrm{FE}$ layer, including a conventional $\mathrm{LSMO} / \mathrm{Alq}_{3} / \mathrm{Co}$ OSV (LSMO-OSV) and a LSMO/STO/Alq $/$ Co OSV (STO-OSV) in which the 5-nm PZT is replaced by 5-nm STO (see Supplementary Figs 6 and 7, and Supplementary Note 5 for detailed MR loops and MR ( $\left.V_{\mathrm{MR}}\right)$ profiles). This indicates that the hysteretic behaviour of the MR in FE-OSV is tied to the presence of PZT. The distinctly different behaviour between the FE-OSV and the PZT-free OSVs (LSMO-OSV and STO-OSV) also allow us to exclude the possibility of resistive bistability mechanism caused by the existence of the trap states or current conduct path inside the $\mathrm{Alq}_{3}$ layer ${ }^{20,21}$, as otherwise similar MR behaviour should be observed in both types of devices. We have also performed measurements on a LSMO/PZT/Co magnetic tunnel junction (FE-MTJ, see Supplementary Fig. 8). Although $V_{\operatorname{MAX}}$

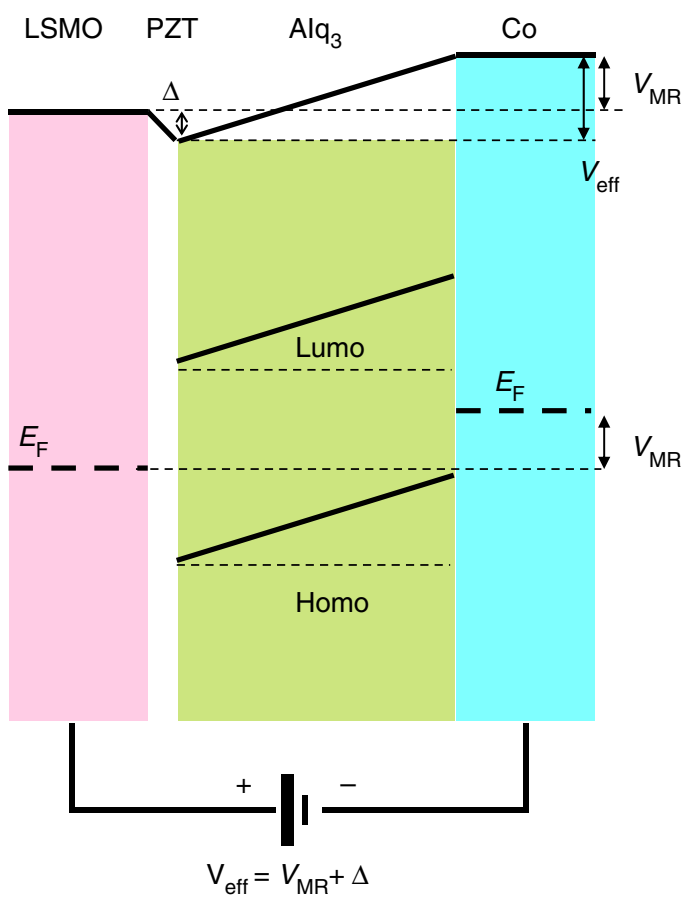

Figure 3 | Model of the hysteretic behaviour in FE-OSV. The relation between the effective bias on the spacer $\mathrm{Alq}_{3}\left(V_{\text {eff }}\right)$, the applied bias at measurement $\left(V_{M R}\right)$ and the vacuum level shift $(\Delta)$ by the remanent dipole moments of the FE PZT layer, is $V_{\text {eff }}=V_{M R}+\Delta$.

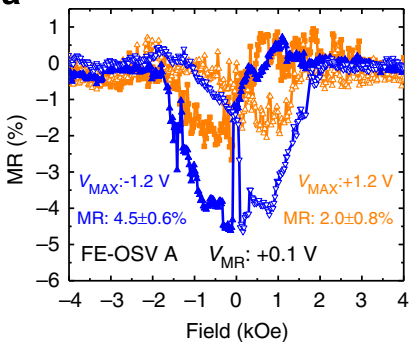

d

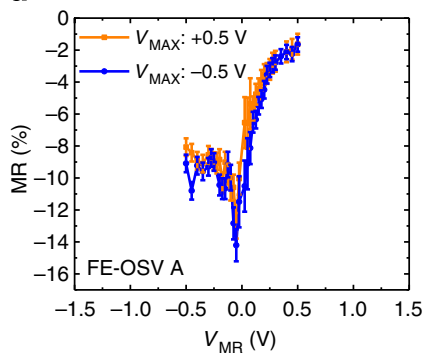

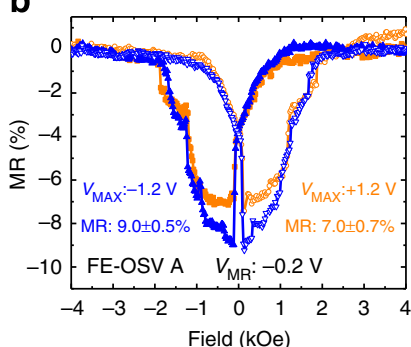

e

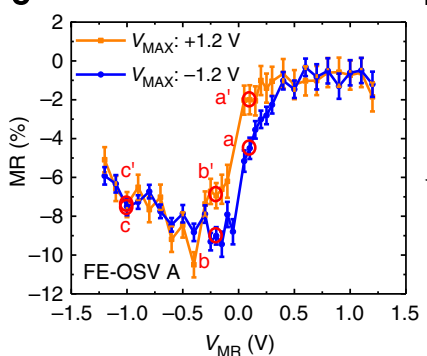

c

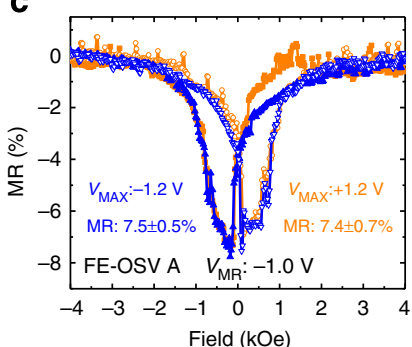

f

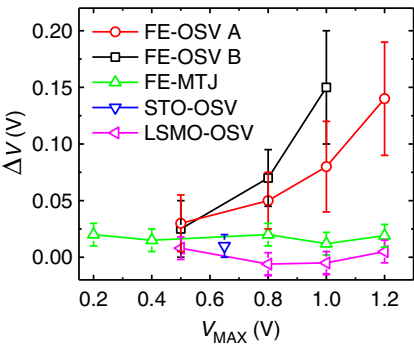

Figure 2 | Hysteretic behaviour of the MR at $\boldsymbol{T}=\mathbf{1 1} \mathbf{K}$. (a-c) MR scans in the as-grown state of a FE-OSV device (PZT in 'up' polarization) with the same applied bias $\left(V_{M R}\right)$ but different initial ramping voltage $\left(V_{M A X}\right)$. Here, LSMO is treated as the anode. The switching fields of FE-OSV are confirmed by superconducting quantum interference device measurements (see Supplementary Fig. 3 and Supplementary Note 3). (d,e) MR $\left(V_{M R}\right)$ profiles taken at two different values of positive (orange) and negative (blue) $V_{M A X} . \Delta V$ represents the shift of MR $\left(V_{M R}\right)$ profiles, and $\mathbf{a}^{\prime} / \mathbf{a}, \mathbf{b}^{\prime} / \mathbf{b}$ and $\mathbf{c}^{\prime} / \mathbf{c}$ mark the positions at which the six MR loops in a-c are taken $\left(V_{\text {MAX: }}+1.2 \mathrm{~V}\right.$ and $-1.2 \mathrm{~V}$ ). (f) Dependence of $\Delta V$ on $V_{\text {MAX. }} \Delta V$ for both FE-OSV devices ( $A$ and $B$ ) increases with increasing $V_{M A X}$, while the three types of control devices (FE-MTJ, STO-OSV and LSMO-OSV) do not show any significant $\Delta V$. The error bars represent the uncertainty of the MR measurements including both systematic error (estimated from the asymmetry in the MR loops) and the statistical error (see Supplementary Fig. 4 and Supplementary Note 4). 
affects the MR $\left(V_{\mathrm{MR}}\right)$ profile, which confirms the results of Pantel et $a .^{22}$ and Valencia et al. ${ }^{23}$, it does not induce any shift of the $\mathrm{MR}\left(V_{\mathrm{MR}}\right)$ profile along the $V_{\mathrm{MR}}$ axis. The comparison of the MR $\left(V_{\mathrm{MR}}\right)$ profiles of the FE-OSV and the FE-MTJ suggests that the effective voltage generated by the dipole of the PZT (instead of the interfacial bonding) is responsible for the hysteretic behaviour of the MR in the FE-OSV. The FE-MTJ does not show hysteretic behaviour of MR, because no direct dipole exists on the surface of the PZT due to the screening effect from the two metal electrodes in direct contact with the PZT layer.

A schematic model is illustrated in Fig. 3 to explain this hysteretic behaviour of MR in the FE-OSV. When a $V_{\text {MAx }}$ is applied to the junction, the polarization of the PZT gets modified. Owing to the dipole moment created by the electric polarization of PZT, the effective voltage $V_{\text {eff }}$ applied on the $\mathrm{Alq}_{3}$ layer equals $V_{\mathrm{MR}}+\Delta$, where $\Delta$ is the vacuum level shift caused by the remnant dipole moment of PZT. When a positive (negative) $V_{\text {MAX }}$ is applied, $\Delta$ becomes larger (smaller), which explains why the MR $\left(V_{\mathrm{MR}}\right)$ profile can be shifted by $V_{\mathrm{MAX}}$.

Switch of the MR sign. Remarkably, a reversal of the polarization of the PZT layer leads to a sign change of the MR in the FE-OSV. After measuring the MR and MR $\left(V_{M R}\right)$ profile (Fig. $\left.4 a-c\right)$ for the FE-OSV device B (PZT layer in the as-grown state), we measured polarization-voltage dependence up to $\pm 5 \mathrm{~V}$. The measurement ended at $-5.0 \mathrm{~V}$ to pole the PZT to the 'down' state. The MR measurements of the FE-OSV device after this treatment are shown in Fig. 4d,e,g,h. The shape of the MR $\left(V_{\mathrm{MR}}\right)$ profile changes dramatically, as shown in Fig. 4i. In particular, the sign of the MR changes from negative (Fig. 4a,b) to positive (Fig. 4d,g,h) for a certain range of $V_{\mathrm{MR}}$. A close correlation between the polarization of PZT and the sign of the MR can be identified by comparing the hysteretic behaviour of the $\mathrm{MR}\left(V_{\mathrm{MR}}\right)$ profile and the possible minor polarization-voltage loop of the PZT, as illustrated in Fig. 4f, that is, when the polarization of PZT is negative enough (more 'down' state), the MR becomes positive.

The physical origin of MR sign of the $\mathrm{LSMO} / \mathrm{Alq}_{3} / \mathrm{Co}$ junctions has been studied previously by Barraud et al. using nanoindentation-based devices. It was argued that first, the spin polarization alignment $P^{\star}$ at the $\mathrm{Co} / \mathrm{Alq}_{3}$ interface is positive when electrons move from Co to $\mathrm{Alq}_{3}$, that is, $P^{\star}\left(\mathrm{Alq}_{3} \rightarrow \mathrm{Co}\right)>0$ where the arrow indicates the direction of the electric current ${ }^{4,24}$; second, the density of states for $\mathrm{Alq}_{3}$ at the $\mathrm{Alq}_{3} / \mathrm{LSMO}$ interface is spin polarized due to the coupling between the two materials, causing $P^{\star}\left(\mathrm{LSMO} \rightarrow \mathrm{Alq}_{3}\right)<0$ when the $\mathrm{Alq}_{3}$ serves as a diffusive spacer. Following these arguments, Barraud et al. ${ }^{16}$ concluded that the sign of MR at small measurement voltages is determined by the sign of the product of $P^{\star}\left(\mathrm{Alq}_{3} \rightarrow \mathrm{Co}\right)$ and $P^{\star}(\mathrm{LSMO} \rightarrow$ $\mathrm{Alq}_{3}$ ); the result is negative. In the FE-OSV studied here, the a

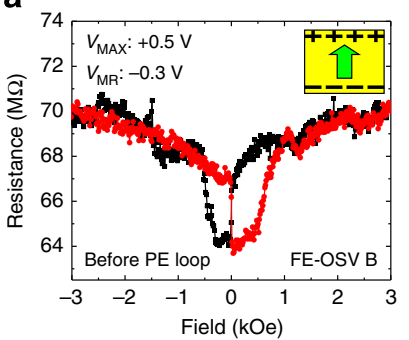

d

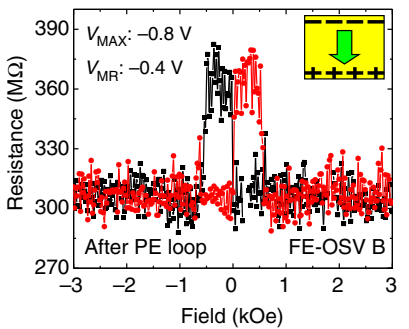

g

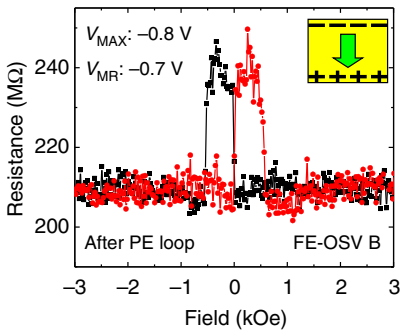

b

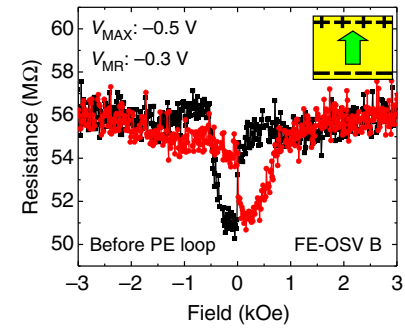

e

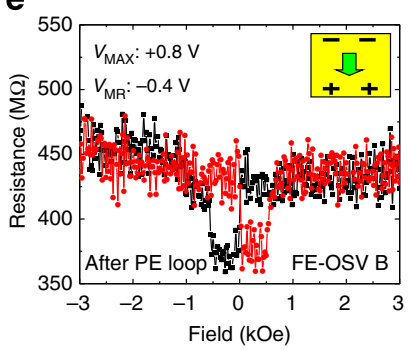

h

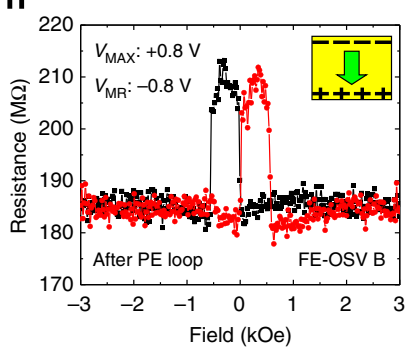

C

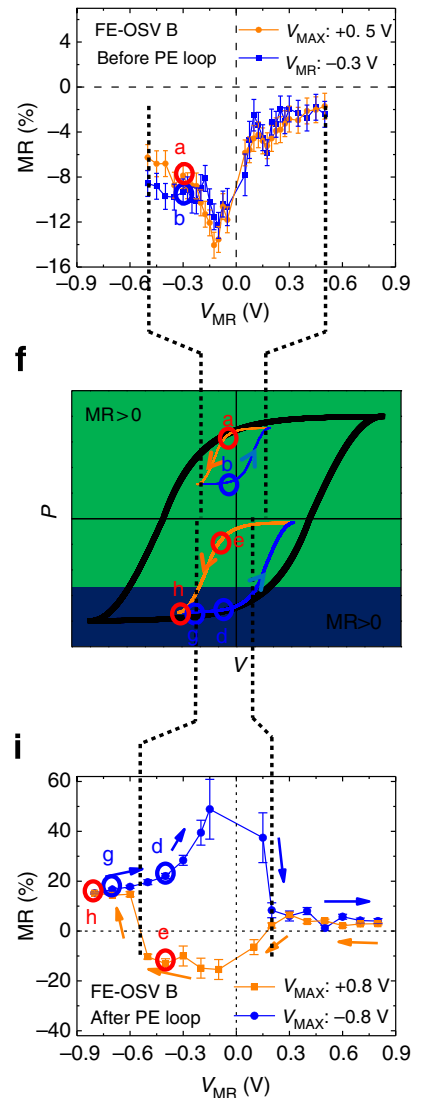

Figure 4 | Reversal of MR sign at $\boldsymbol{T}=\mathbf{1 1} \mathbf{K}$. (a,b) $M R$ scans for the 'as-grown' state of the FE-OSV device $B$ acquired at $V_{M R}=-0.3 V / V_{M A X}=+0.5 V$ and $V_{M R}=-0.3 \mathrm{~V} / V_{M A X}=-0.5 \mathrm{~V}$, respectively. (c) $M R\left(V_{M R}\right)$ profiles at $V_{M A X}=+0.5 \mathrm{~V}$ (orange) and $-0.5 \mathrm{~V}$ (blue). (d,e,g,h) MR scans taken when the polarization of the PZT is reversed. (f) Comparison of the MR $\left(V_{M R}\right)$ profile and the polarization-voltage loop, illustrating the correlation between the sign of $M R$ and the polarization of the PZT. The asymmetric regions of $M R<0$ and $M R>0$ in polarization-voltage loop indicate that the reversal of MR only occurs at higher degree of polarization in the 'down' state of PZT. (i) MR( $\left.V_{M R}\right)$ profiles at $V_{\text {MAx: }} \pm 0.8 \mathrm{~V}$ when the polarization of the PZT is reversed. The red (blue) circles indicate the $V_{M R}$ values at which the MR loops $(\mathbf{a}, \mathbf{b}, \mathbf{d}, \mathbf{e}, \mathbf{g}, \mathbf{h})$ are taken with positive (negative) $V_{M A X}$. The error bars are estimates using the same analysis as that in Fig. 2. 
$P^{*}\left(\mathrm{Alq}_{3} \rightarrow \mathrm{Co}\right)>0$ can be assumed similarly; the polarization alignment $P^{*}$ between LSMO and $\mathrm{Alq}_{3}$ is not changed by inserting a thin layer $(5 \mathrm{~nm})$ of as-grown PZT or STO, but can be changed by poling the PZT layer, according to the behaviour of the observed MR. This novel feature in FE-OSV must be due to the more complex LSMO/PZT $\rightarrow \mathrm{Alq}_{3}$ interface. We propose the following model based on the relative energy level alignment between $\mathrm{Alq}_{3}$ and LSMO to understand the switching of MR sign in FE-OSV.

Here we consider mainly the hole transport, because for $\mathrm{Alq}_{3}$ the energy difference between the highest occupied molecular orbital (HOMO) and Fermi levels of the two metallic LSMO and Co electrodes are much smaller than that between the lowest unoccupied molecular orbital and the Fermi levels $2,18,25$. As shown in Fig. 5a, when the polarization of the PZT layer is pointing 'up' (the as-grown state) or zero (the same case as for STO), the hole injection from the LSMO electrode for positive $V_{\mathrm{MR}}$ is from the Fermi level (which lies in the spin majority band) of LSMO to the HOMO of $\mathrm{Alq}_{3}$, which is the same as in the LSMO-OSV. Therefore, $P^{\star}\left(\mathrm{LSMO} / \mathrm{PZT} \rightarrow \mathrm{Alq}_{3}\right)<0$, corresponding to a negative MR considering $P^{\star}\left(\mathrm{Alq}_{3} \rightarrow \mathrm{Co}\right)>0$ as discussed above ${ }^{2,4}$. When the polarization of the PZT layer is pointing 'down', the $\mathrm{HOMO}$ of $\mathrm{Alq}_{3}$ is shifted up due to the dipole moment of PZT. Therefore, another state of the LSMO with opposite spin polarization may be accessible (Fig. 5b). Hence, $P^{\star}\left(\mathrm{LSMO} / \mathrm{PZT} \rightarrow \mathrm{Alq}_{3}\right)$ changes the sign and becomes positive, corresponding to a reversed, positive MR.

Our proposed model relies on two key assumptions: (1) the dipole moment of the PZT layer shifts the $\mathrm{Alq}_{3} \mathrm{HOMO}$ level and (2) the shift of the $\mathrm{Alq}_{3} \mathrm{HOMO}$ level results in a shift of the initial state of LSMO for the hole injection between energy band of opposite spin polarizations. The first assumption was used by Schultz et al. ${ }^{18}$ to explain the MR sign reversal in a $\mathrm{FeCo} / \mathrm{Alq}_{3} /$ $\mathrm{LiF} / \mathrm{NiFe}$ junction by proposing a shift of the $\mathrm{HOMO}$ of $\mathrm{Alq}_{3}$ due a

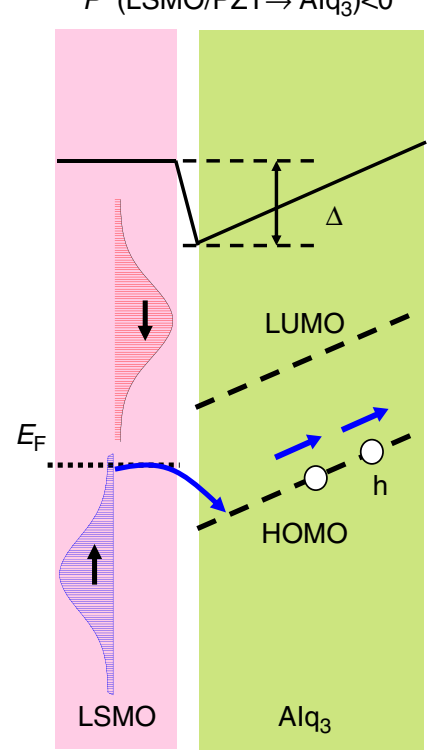

$\mathrm{PZT} \uparrow$

$\mathrm{MR}<0$ b

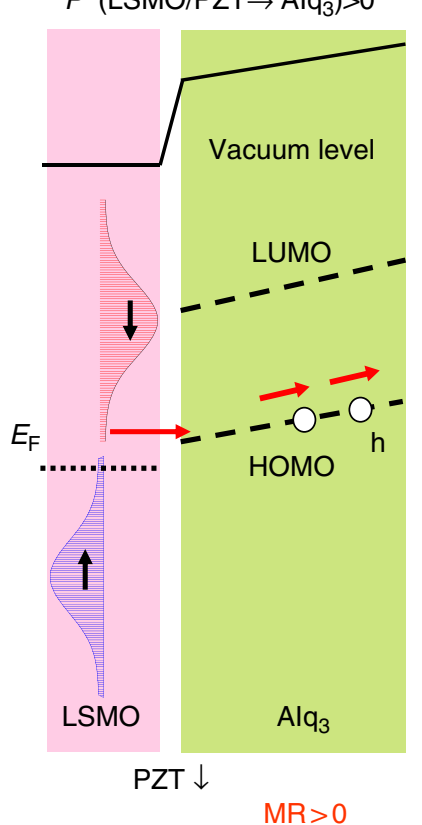

Figure $\mathbf{5}$ | Model of MR sign reversal in FE-OSV. $(\mathbf{a}, \mathbf{b})$ The energy

diagrams of the FE-OSV device when the electric polarization of the PZT is 'up' and 'down', respectively. The white circles represent the injected holes in the device. The blue (red) arrows indicate the injection of spin-polarized holes from the majority (minority) band of LSMO. to the dipole moment of LiF layer. The second assumption can be justified by the half metallicity of LSMO. As illustrated in Fig. 5, the conduction band of LSMO splits into spin majority and minority bands due to the exchange interaction, causing half metallicity because the Fermi level lies within the fully polarized spin majority band ${ }^{26-28}$. Therefore, when the HOMO of $\mathrm{Alq}_{3}$ is shifted up due to the reversal of dipole moment of PZT, the initial state of LSMO for hole injection may change to spin minority band with opposite spin polarization. Note that this reversed dipole moment of PZT needs to be large enough to shift the HOMO level of $\mathrm{Alq}_{3}$ to 'reach' the spin minority band of LSMO and obtain reversed positive MR values. Otherwise, only negative MR values will be observed, as shown in Fig. 4f (also see Supplementary Fig. 9 and Supplementary Note 6).

Besides changing the energy level alignments, switching the electric polarization of the FE layer may also modify the coupling between the FE layer and the magnetic electrode, depending on the detailed electronic structure of the electrode and the nature of the electric polarization of the FE material ${ }^{29-31}$. These effects may also change the spin polarization at the interface between the FE material and the metal electrode $22,23,31$. However, both the magnetic structure of the LSMO and the spin polarization of the PZT are not expected to be affected very much by the electric polarization of the PZT, because of the robust magnetic properties of the $\mathrm{La}_{0.7} \mathrm{Sr}_{0.3} \mathrm{MnO}_{3}$ with given composition (far from the metal-insulator phase boundary) and the large distance between the Mn site from LSMO and Ti sites from PZT at the interface $22,23,30,31$.

Another possible scenario involves the change of carrier type when the energy level alignment between LSMO and $\mathrm{Alq}_{3}$ is changed: the carriers take the path of the HOMO (lowest occupied molecular orbital),that is, hole (electron) transport in $\mathrm{Alq}_{3}$ when the energy levels of $\mathrm{Alq}_{3}$ is shifted 'down' ('up') due to the 'up' ('down') polarization of the PZT layer. This, however, contradicts our experimental observations because in the FE-OSV with the as-grown ('up' polarization) PZT, the MR is negative, the same as that in the STO-OSV or LSMO-OSV without any interfacial layers, suggesting that the carrier type in FE-OSV is most probably holes instead of electrons ${ }^{18,25}$, as in the STO-OSV or LSMO-OSV.

\section{Discussion}

The active control of the energy level alignment between the electrodes and the organic material, manifested here in the active control of the MR, not only carries promises for multistate control of organic spin-valve devices, but will also impact other organic electronic devices, in particular those applied in photovoltaics and solid-state lighting. Specifically, the charge carrier injection efficiency of the organic light emitting diode is determined by the relative alignment between the Fermi energy of the electrode and the energy levels of the organic material ${ }^{32}$. The charge collection efficiency in an organic photovoltaic device also depends on the alignment of the energy levels of the acceptor organic material and the electrode ${ }^{33}$. Therefore, the realization of the active control of the level alignment using an FE interfacial layer demonstrated in this letter may also lead to successful optimization of other organic electronic devices by tailoring the energy landscape of the comprising materials using a tunable interfacial layer.

\section{Methods}

Device fabrication. PZT (5 nm), STO $(5 \mathrm{~nm})$ and LSMO $(30 \mathrm{~nm})$ thin films epitaxially grown on STO (001) substrate by pulsed laser deposition were fabricated into bottom electrodes using conventional wet-etching photolithography ${ }^{11,34}$ The $\mathrm{Alq}_{3}$ (99.995\%, Aldrich) layer (thickness: $65 \mathrm{~nm}$ ) was deposited by thermal evaporation onto a room-temperature substrate in a vacuum chamber with base 
pressure of $5.0 \times 10^{-9}$ Torr. Without breaking the vacuum, the Co $(10 \mathrm{~nm}) / \mathrm{Au}$ $(7 \mathrm{~nm})$ was then deposited by thermal evaporation at substrate temperature of $280 \mathrm{~K}$ to complete the formation of the top electrode in a crossbar configuration through the shadow mask. The thickness of layers were controlled by a quartz thickness monitor. The device area is $\sim 200 \mu \mathrm{m} \times 50 \mu \mathrm{m}$.

Device characterizations. Atomic force microscopy topography images and PFM were taken using a Veeco Dimension 3100 at room temperature. The TEM samples were prepared by a FEI Dual-beam (Focus-Ion-Beam/SEM) system, and highresolution TEM images were taken by a FEI Tecnai transmission electric microscope with beam energy of $200 \mathrm{kV}$. Radiant Premier II FE measuring system was used to detect the polarization-voltage loops of the devices at $50 \mathrm{~Hz}$. Magnetometer measurements were carried out using a Quantum Design superconducting quantum interference device system. Magnetic fields were applied in the plane of the thin film. Transport measurements were carried out using a Quantum Design Physical Property Measurement System (PPMS) combined with a Keithley 2400 source meter at $T=11 \mathrm{~K}$. Magnetic fields were applied in the plane of the thin film. The MR is defined as: $M R=\left(R_{\text {antiparallel }}-R_{\text {parallel }}\right) / R_{\text {parallel, }}$, where $R_{\text {antiparallel }}$ is the junction resistance in the antiparallel magnetic configuration and $R_{\text {parallel }}$ is the resistance at the parallel configuration. The detailed MR measurement protocols are shown in Supplementary Fig. 2 and Supplementary Note 2.

\section{References}

1. Dediu, V., Murgia, M., Matacotta, F. C., Taliani, C. \& Barbanera, S. Room temperature spin polarized injection in organic semiconductor. Solid State Commun. 122, 181-184 (2002).

2. Xiong, Z. H., Wu, D., Vardeny, Z. V. \& Shi, J. Giant magnetoresistance in organic spin-valves. Nature 427, 821-824 (2004).

3. Fert, A. Nobel Lecture: Origin, development, and future of spintronics. Rev. Mod. Phys. 80, 1517-1530 (2008).

4. Dediu, V. et al. Room-temperature spintronic effects in $\mathrm{Alq}_{3}$-based hybrid devices. Phys. Rev. B 78, 115203 (2008).

5. Dediu, V. A., Hueso, L. E., Bergenti, I. \& Taliani, C. Spin routes in organic semiconductors. Nat. Mater. 8, 707-716 (2009).

6. Wang, F. \& Vardeny, Z. V. Recent advances in organic spin-valve devices. Synth. Met. 160, 210-215 (2010).

7. Schoonus, J. J. et al. Magnetoresistance in hybrid organic spin valves at the onset of multiple-step tunneling. Phys. Rev. Lett. 103, 146601 (2009).

8. Nguyen, T. D. et al. Isotope effect in spin response of $\pi$-conjugated polymer films and devices. Nat. Mater. 9, 345-352 (2010).

9. Drew, A. J. et al. Direct measurement of the electronic spin diffusion length in a fully functional organic spin valve by low-energy muon spin rotation. Nat. Mater. 8, 109-114 (2009).

10. Cinchetti, M. et al. Determination of spin injection and transport in a ferromagnet/organic semiconductor heterojunction by two-photon photoemission. Nat. Mater. 8, 115-119 (2009).

11. Sun, D. et al. Giant magnetoresistance in organic spin valves. Phys. Rev. Lett. 104, 236602 (2010)

12. Zhan, Y. Q. et al. The role of aluminum oxide buffer layer in organic spinvalves performance. Appl. Phys. Lett. 94, 53301-53303 (2009).

13. Zhan, Y. Q. et al. Energy level alignment and chemical interaction at $\mathrm{Alq}_{3} / \mathrm{Co}$ interfaces for organic spintronic devices. Phys. Rev. B 78, 45208 (2008).

14. Grobosch, M., Dorr, K., Gangineni, R. B. \& Knupfer, M. Energy level alignment and injection barriers at spin injection contacts between $\mathrm{La}_{0.7} \mathrm{Sr}_{0.3} \mathrm{MnO}_{3}$ and organic semiconductors. Appl. Phys. Lett. 92, 23302-23303 (2008).

15. Zhan, Y. Q. et al. Alignment of energy levels at the $\mathrm{Alq}_{3} / \mathrm{La}_{0.7} \mathrm{Sr}_{0.3} \mathrm{MnO}_{3}$ interface for organic spintronic devices. Phys. Rev. B 76, 45406 (2007).

16. Barraud, C. et al. Unravelling the role of the interface for spin injection into organic semiconductors. Nat. Phys. 6, 615-620 (2010).

17. Sanvito, S. Molecular spintronics: the rise of spinterface science. Nat. Phys. 6, 562-564 (2010)

18. Schulz, L. et al. Engineering spin propagation across a hybrid organic/inorganic interface using a polar layer. Nat. Mater. 10, 39-44 (2011).

19. Pacchioni, G. Two-dimensional oxides: multifunctional materials for advanced technologies. Chem. A Eur. J. 18, 10144-10158 (2012).

20. Prezioso, M. et al. Electrically programmable magnetoresistance in multifunctional organic-based spin valve devices. Adv. Mater. 23, 1371-1375 (2011).

21. Prezioso, M. et al. A single-device universal logic gate based on a magnetically enhanced memristor. Adv. Mater. 25, 534-538 (2013).

22. Pantel, D., Goetze, S., Hesse, D. \& Alexe, M. Reversible electrical switching of spin polarization in multiferroic tunnel junctions. Nat. Mater. 11, 289-293 (2012).
23. Valencia, S. et al. Interface-induced room-temperature multiferroicity in $\mathrm{BaTiO}_{3}$. Nat. Mater. 10, 753-758 (2011).

24. Santos, T. S. et al. Room-temperature tunnel magnetoresistance and spinpolarized tunneling through an organic semiconductor barrier. Phys. Rev. Lett. 98, 16601 (2007).

25. Nguyen, T. D., Ehrenfreund, E. \& Vardeny, Z. V. Spin-polarized light-emitting diode based on an organic bipolar spin valve. Science 337, 204-209 (2012).

26. Park, J.-H. et al. Direct evidence for a half-metallic ferromagnet. Nature 392, 794-796 (1998).

27. Bowen, M. et al. Nearly total spin polarization in $\mathrm{La}_{2 / 3} \mathrm{Sr}_{1 / 3} \mathrm{MnO}_{3}$ from tunneling experiments. Appl. Phys. Lett. 82, 233-235 (2003).

28. Park, J.-H. et al. Magnetic properties at surface boundary of a half-metallic ferromagnet $\mathrm{La}_{0.7} \mathrm{Sr}_{0.3} \mathrm{MnO}_{3}$. Phys. Rev. Lett. 81, 1953-1956 (1998).

29. López-Encarnación, J. M., Burton, J. D., Tsymbal, E. Y. \& Velev, J. P. Organic multiferroic tunnel junctions with ferroelectric poly(vinylidene fluoride) barriers. Nano Lett. 11, 599-603 (2010).

30. Burton, J. D. \& Tsymbal, E. Y. Prediction of electrically induced magnetic reconstruction at the manganite/ferroelectric interface. Phys. Rev. B 80, 174406 (2009).

31. Gracia, V. et al. Ferroelectric control of spin polarization. Science 327, 1106-1110 (2010).

32. Shen, Y., Hosseini, A. R., Wong, M. H. \& Malliaras, G. G. How to make ohmic contacts to organic semiconductors. ChemPhysChem 5, 16-25 (2004).

33. Yuan, Y. et al. Efficiency enhancement in organic solar cells with ferroelectric polymers. Nat. Mater. 10, 296-302 (2011).

34. Lee, H. N. et al. Suppressed dependence of polarization on epitaxial strain in highly polar ferroelectrics. Phys. Rev. Lett. 98, 217602 (2007).

\section{Acknowledgements}

This study was supported by the National Basic Research Program of China (973 Program) under the grant numbers 2011CB921800, 2013CB932901 and 2014CB921104; National Natural Science Foundation of China (91121002 and 11274071); Shanghai Municipal Natural Science Foundation (11ZR1402600); China Postdoctoral Science Foundation (2013M540321); the Wuhan National High Magnetic Field Center (WHMFCKF2011008) (M.F., L.Y., Y.W., W.Y. and J.S.). We also acknowledge the funding support of U.S. Department of Energy, Basic Energy Sciences, Materials Sciences and Engineering Division (D.S., X.X., L.J., H.N.L., P.C.S. and T.Z.W.) and the U.S Department of Energy, Basic Energy Sciences, Scientific User Facilities Division (X.G.Z. and Z.G.), the US DOE grant DE-SC0002136 (H.G. and J.S.).

\section{Author contributions}

D.S. and X.X. designed and supervised the experiments. D.S., M.F., X.X. and L.F. prepared the experimental setup. D.S. and M.F. carried out the transport measurement. M.F., D.S., H.G., Y.W. and W.Y. carried out the device preparations. M.F., Y.W. and W.Y. carried out the measurements of TEM imaging, PE loops and magnetic properties L.J and H.N.L. were responsible for growing PZT, STO, LSMO films and PFM measurements. D.S., M.F., X.X, H.W., P.C.S., T.Z.W. and Z.G. carried out the data analysis. X.X. and X.G.Z. contributed to the theoretical modelling for the interpretation. J.S. was responsible for project planning, group managing and the final writing. All authors contributed to the physical understanding, data analysis and manuscript preparation.

\section{Additional information}

Supplementary Information accompanies this paper at http://www.nature.com/ naturecommunications

Competing financial interests: The authors declare no competing financial interests.

Reprints and permission information is available online at http://npg.nature.com/ reprintsandpermissions/

How to cite this article: Sun, D. et al. Active control of magnetoresistance of organic spin valves using ferroelectricity. Nat. Commun. 5:4396 doi: 10.1038/ncomms5396 (2014).

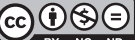

This work is licensed under a Creative Commons AttributionNonCommercial-NoDerivs 4.0 International License. The images or other third party material in this article are included in the article's Creative Commons license, unless indicated otherwise in the credit line; if the material is not included under the Creative Commons license, users will need to obtain permission from the license holder to reproduce the material. To view a copy of this license, visit http:// creativecommons.org/licenses/by-nc-nd/4.0/ 